Graham C. Holt

\title{
Cloaking devices: progress with metamaterials
}

ABSTRACT. Since the practical realization of negative refractive index materials in 2001, considerable progress has been made in both designing the materials and extending the range of the electromagnetic spectrum that may be manipulated. This paper reviews progress, especially in the field of visible light and the applications to cloaking devices, lenses and switches.

Nanotechnology Perceptions 10 (2014) 61-67 